\title{
SIMULATION STUDY OF WAKE FIELD EXCITATION IN INTERACTION OF INTENSE LASER AND MAGNETIZED PLASMA: HALF-SINE PULSE SHAPE (HSPS) AND TRAPEZOID PULSE SHAPE (TPS)
}

\author{
A. Rahimian and H. Zahed \\ Department of Physics, Sahand University of Technology, P. O. Box 51335-1996, Tabriz, Iran \\ E-mail: a_rahimian@sut.ac.ir
}

Received 22 October 2016; revised 5 January 2017; accepted 16 March 2017

\begin{abstract}
We have conducted particle-in-cell (PIC) simulations of a linearly polarized intensive laser pulse with two different envelopes propagating through a homogeneous fully ionized cold plasma. It is shown that the amplitude of the wake field depends on laser wavelength, pulse duration, electron number density and envelope shape. We have also simulated the effect of applying a longitudinal magnetic field on the wake field excitation process. It is observed that magnetic field enhances the wake field and increases its intensity in all cases. Our results are in agreement with the analytical results presented by Askari and Shahidani [Opt. Laser Technol. 45, 613-619 (2013)] and can help choosing the optimum values of affecting laser and plasma parameters in order to reach high accelerating wake electric fields.
\end{abstract}

Keywords: laser pulse, wake field excitation, particle-in-cell (PIC), magnetized plasma

PACS: 02.60.Cb, 52.25.Xz, 52,38.-r

\section{Introduction}

As laser beams reach higher intensities, laser plasma interactions draw higher interests since past few decades, because of the relevance to laser driven fusion, plasma based accelerators, X-ray lasers and other applications [1-3]. A great deal of efforts have been made to obtain high accelerating fields using short $(<1 \mathrm{ps})$ ultrahigh intensity $\left(>10^{18} \mathrm{~W} / \mathrm{cm}^{2}\right)$ lasers in an underdense plasma. Acceleration of the charged particles has very important applications in physical sciences. For example, particle physics, nuclear physics, and astrophysics. It has been an interesting subject for the both experimental and theoretical researches. Also the important industrial and medicinal applications should be enumerated [4]. The ponderomotive force of a short, ultrahigh intensity laser pulse, which is proportional to the negative gradient of the laser intensity, excites the plasma waves assuming the laser pulse maintains its shape. The electrons are pushed away from the laser pulse area and separated from the ions, which are stationary because of their much higher mass. This charge separation forms a travelling electric field behind the laser pulse. That is, the wake field. This wake field is a large longitudinal accelerating electric field. It can accelerate charged particles to higher energies than that of conventional linear accelerators in much shorter distances. The magnitude of wake field can be more than $100 \mathrm{GV} / \mathrm{m}$. The accelerating distance is about plasma wavelength, i. e. 10$30 \mu \mathrm{m}$, for plasma densities about $10^{18}-10^{20} \mathrm{~cm}^{-3}$. Laser plasma accelerators can generate high quality electron beams for which the energy distribution is quasi-monoenergetic [5-7]. There are several acceleration schemes in plasma based accelerators which work on the same principle. These schemes 
are laser wake field accelerator (LWFA) [8], plasma wake field accelerator (PWFA) [9], plasma beat wave accelerator (PBWA) [10-12], self-modulated laser wake field accelerator (SM-LWFA) [13-15, and wake field accelerators driven by multiple electron or laser pulses [16]. Among these schemes, the LWFA was introduced for the first time. Since the publication of the LWFA idea for the first time [8], a noticeable progress in both experimental [ ] 17, 18] and theoretical physics [3] has been made. Leemans et al. have reported generating $\mathrm{GeV}$ electron beams in their experiment [18]. This energy level is almost equal to that (the level) of conventional RF linacs. Nevertheless, solving all physical problems in order to reach future high gradient accelerators is really overwhelming. Controlling the charge and energy of the electron beam and improving its quality is one of the most important subjects. Electron acceleration via laser plasma interaction is rich in nonlinear effects, such as fast electron and ion generation [19, 20], which is a result of the presence of ultra-intense electromagnetic fields in the process. The effect of magnetic fields on nonlinear plasma dynamics could not be ignored. A jet of fast electrons flowing along the laser propagation direction [21] or a nonlinear current of the plasma electrons [22] is usually used to generate magnetic fields. Generation of magnetic fields up to $70 \mathrm{~T}$ using the technology of pulsed magnets is also reported [23]. Ultra-intense magnetic fields have a significant effect on particle transport, propagation of laser pulses and laser beam self-focusing. Therefore, the propagation of laser pulse in magnetized plasma has been an interesting subject for many researchers. Shukla et al. have investigated the nonlinear propagation of an electromagnetic wave in a strongly magnetized electron-positronion plasma. They have found that the generation of wake fields depends to some extent on the presence of ion species and external magnetic field [24]. Xiong et al. have studied the influence of arbitrary magnetic field on self-focusing of laser pulse propagating in magnetized plasma [25]. They reported that there is a different effect on self-focusing corresponding to a different angle and intensity. Krasovitskii et al. have studied the interaction of powerful laser pulse with plasma and generated wake field in the presence of an external magnetic field [26]. They have shown that the transferred energy to electron oscillations in the wake field is different for various angles between the direction of laser pulse propagation and magnetic field. Also, Jha et al. have studied the evolution of the spot size of intense laser beam propagating in axially magnetized plasma [27]. They have obtained the critical power for nonlinear self-focusing of the beam.

It is obvious that the effects of the presence of an external magnetic field on laser plasma nonlinear processes are sophisticated. Hence, different aspects of this subject still need to be investigated. One of the important and efficient tools in investigations is numerical simulations. Modelling a plasma-based accelerator needs a code which could be able to simulate the driver evolution, the wake generation and the acceleration of particles. Among various methods, particle based models can do this properly because the trajectories of particles should be followed in their self-consistent fields. Fluid descriptions cannot model a highly nonlinear wake excitation process and resulting particle trajectories. The most straightforward particle based model is the fully explicit particle-in-cell (PIC) algorithm [28]. The PIC method simulates the selfconsistent interaction dynamics of particles (electrons and ions) and electromagnetic fields in a 1D, $2 \mathrm{D}$ or $3 \mathrm{D}$ mesh [29].

\section{Formulation and initial set up}

In this paper, 2 and 1/2-dimensional particle-incell simulations are carried out to study the wake field produced by propagation of an intense linearly polarized laser pulse with a transverse Gaussian profile and two different temporal envelopes (Figs. 1 $(a, b))$ : Half-Sine shape and Trapezoid shape.

We have also studied the effect of the presence of a magnetic field along the laser propagation direction on the wake field excitation. The ambient plasma is homogeneous, cold and pre-ionized with a linear density ramp of the form $n(x)=2 \mathrm{a} x+\mathrm{b}$, where $n(0)=n^{1}, n(L)=n^{2}$ and $L$ is the ramp length. The driving laser beam is in the TM-mode (S-polarized) with a wavelength of $\lambda=1.0 \mu \mathrm{m}$ corresponding to a frequency of $\omega_{L}=2 \pi c / \lambda=1.88 \times 10^{15} \mathrm{~Hz}$, where $c$ is the light speed in vacuum. The plasma bulk density $\left(n_{0}\right)$ is $5 \times 10^{24} \mathrm{~m}^{-3}$ which corresponds to the plasma wavelength of $15 \mu \mathrm{m}$ via $\lambda_{P}(\mu \mathrm{m})-3.3 \times 10^{10} / \sqrt{n_{0}\left(\mathrm{~cm}^{-3}\right)}$. The ions are assumed to be stationary forming a uniform background of 
(a)

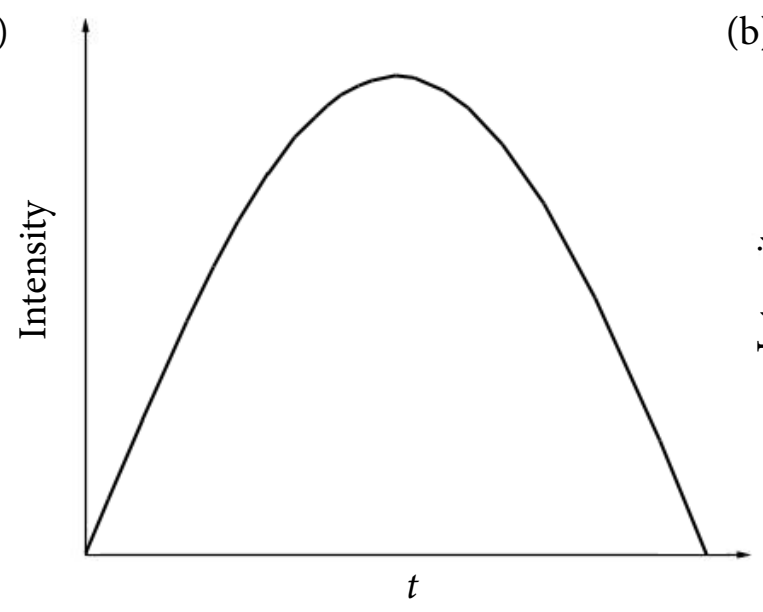

(b)

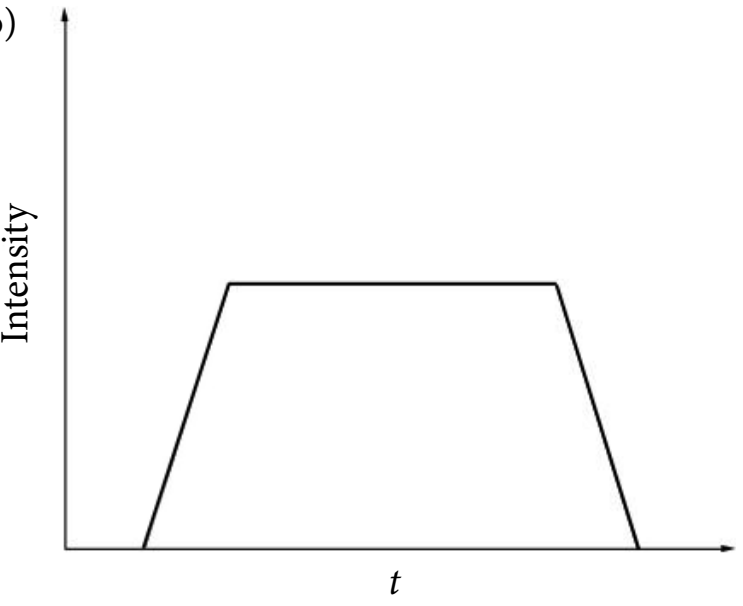

Fig. 1. Profiles of laser pulse: (a) half-sine shape and (b) trapezoid shape.

charges. The intensity of laser pulse employed in this simulation is $I_{0}=3 \times 10^{22} \mathrm{~W} / \mathrm{m}^{2}$ which falls into the relativistic regime. Laser pulse intensity is related to the dimensionless laser strength parameter $a_{0}$ by $a_{0}=0.85 \lambda(\mu \mathrm{m}) \sqrt{I\left(10^{22} \mathrm{~W} / \mathrm{m}^{3}\right)}$. So, the above $I_{0}$ and $\lambda$ values yield $a_{0}=1.62$. The electron motion is highly relativistic when $a_{0} \geq 1$. This relativistic effect sets the stage for self-focusing [30]. The general expression for the index of refraction in a plasma is given by $\eta_{r}-1-\frac{\omega_{P}}{2 \omega_{L}^{2}} \frac{n(r)}{n_{0} \gamma(r)}$, where $\gamma(r)$ is the relativistic factor, $n(r)$ is the radial density (cylindrical) profile, $\omega_{P}$ is the plasma electron frequency prior to the passage of laser pulse: $\omega_{P}=\left(4 \pi n_{0} e^{2} / m_{e}\right)^{1 / 2}$ and $\gamma-\gamma_{\perp}=\left(1+a^{2}\right)^{1 / 2}$. Here, $\mathbf{a}^{2}=\mathbf{a} \cdot \mathbf{a}$ and $\mathbf{a}$ is the normalized vector potential of the laser field: $\mathbf{a}=e \mathbf{A}$ / $m_{e} c^{2}$, where $e$ is the fundamental charge and $m_{e}$ is the electron mass. A laser intensity profile peaked on the axis $\frac{\partial a^{2}}{\partial r}<0$ leads to $\frac{\partial \eta_{r}}{\partial r}<0$ which implies that the phase velocity along the propagation axis is less than it is off-axis. This causes the laser pulse fronts to curve such that the beam focuses towards the axis. Other considered laser parameters are the full width at half maximum (FWHM) of $12 \mu \mathrm{m}$ (or laser pulse duration of $\tau=40 \times 10^{-15} \mathrm{~s}$ ) and the spot size of $s_{0}=15 \mu \mathrm{m}$. In this simulation we consider a plasma box with the grid size of $\left(N_{x}, N_{y}\right)=(750,256)$ equal to the $75 \mu \mathrm{m} x$ direction length and $256 \mu \mathrm{m} y$ direction length. Each cell contains 6 particles. The time step meets the Courant-Levy stability criterion $\Delta t \leq \frac{1}{c}\left(\sum_{i} \frac{1}{\left(\Delta x_{i}\right)^{2}}\right)$ and the program runs for 1500 time steps.
The laser pulse is launched from the left boundary and propagates along the $x$ direction. Other boundaries are considered to be conductors.

We used the conventional PIC algorithm in our simulation which is briefly described in the following. First, the simulation domain is divided into spatial grids using the Cartesian geometry. Then, the plasma particles are loaded onto the grids and their charges are assigned to the surrounding nodes (using first-order weighting) from which currents are calculated. These source terms are employed to obtain the electric and magnetic fields via discrete Maxwell equations (SI) [31],

$$
\nabla \times \mathbf{E}=-\frac{\partial \mathbf{B}}{\partial t}
$$

and

$$
\nabla \times \mathbf{B}=\mu_{0} \varepsilon_{0} \frac{\partial \mathbf{E}}{\partial t}+\mu_{0} \mathbf{J},
$$

where $\mathbf{E}$ and $\mathbf{B}$ are defined on the Yee-mesh [32]. In order to make sure that the continuity equation $\left(\frac{\partial \rho}{\partial t}+\nabla \mathbf{J}=0\right)$ is observed, the current density $\mathbf{J}$ is calculated using a charge-conservation scheme (CCS). Thereafter, the updated $\mathbf{E}$ and $\mathbf{B}$ fields are used to push the particles into new positions and velocities by employing the relativistic Lorentz equation of motion:

$$
\frac{\mathrm{dP}}{\mathrm{d} t}=q\left(\mathbf{E}+\frac{\mathbf{P}}{\gamma} \times \mathbf{B}\right) .
$$

$\mathbf{P}=\gamma \mathbf{V}$ is the relativistic particle momentum where $\gamma$ is the relativistic factor. Here, the Boris 
scheme is used [33] which is comprised of three steps: half acceleration, rotation and half acceleration. The difference form of Eq. (3) is

$$
\begin{aligned}
& \frac{\mathbf{P}^{t+1 / 2}-\mathbf{P}^{t-1 / 2}}{\Delta t}=q\left(\mathbf{E}^{t}+\frac{\mathbf{P}^{t+1 / 2}+\mathbf{P}^{t-1 / 2}}{2 \gamma^{t}} \times \mathbf{B}^{t}\right) \\
& \mathbf{P}^{-}=\mathbf{P}^{t-^{1 / 2}}-q \mathbf{E}^{t} \frac{\Delta t}{2} \text { (half acceleration) } \\
& \mathbf{P}^{+}=\left(\begin{array}{lll}
1-S_{z} T_{z}-S_{y} T_{y} & S_{y} T_{x}-S_{z} & S_{z} T_{x}-S_{y} \\
S_{x} T_{y}-S_{z} & 1-S_{x} T_{x}-S_{z} T_{z} & S_{z} T_{y}+S_{x y} \\
S_{x} T_{z}+S_{y} & S_{z} T_{z}-S_{x} & 1-S_{x} T_{x}-S_{y} T_{y}
\end{array}\right) \\
& \mathbf{P}^{t t^{-1 / 2}}=\mathbf{P}^{+}+q \mathbf{E}^{t} \frac{\Delta t}{2} \text { (hotation) }
\end{aligned}
$$

Here

$$
\mathbf{T}=\frac{\Delta t}{2 \gamma^{t}} \mathbf{B}^{t}, \mathbf{S}=\frac{2 \mathbf{T}}{1+\mathbf{T}^{2}} \text { and } \gamma=\sqrt{1+\mathbf{P}^{ \pm^{2}}}
$$

These new momentum of particles are used to advance positions. This cycle is repeated for as many time steps as required.

\section{Effect of electron number density}

Electron number density plays an important role in laser plasma interaction. It affects the properties of wake field significantly. In order to study the effect of this parameter, the amplitude of wake field, $E_{\mathrm{wP}}$ for each envelope shape is plotted in terms of $n_{0}$ in Figs. 2 and 3 for two different laser parameters: wavelength and pulse duration, respectively. In Figs. $2(a-c)$ and Figs. $2(d-f)$, the curves are plotted for $\lambda$ values of $0.8,1.1$ and $1.4 \mu \mathrm{m}$, respectively. It should be mentioned that hereafter the highest presented value of $E_{w f}$ in each figure will be named maximum. It can be seen from Fig. 2 that by increasing $\lambda$, the maximum value of $E_{\mathrm{wf}}$ rises and occurs at higher $n_{0}$ values. This behaviour is in complete agreement with Fig. 1(b) in Askari and Shahidani [34. Also, $E_{\text {wf }}$ has higher intensities for HSPS compared to TPS. The maximum magnitudes of $E_{\mathrm{wf}}$ for different $\lambda$ values are shown in Table 1. When the magnetic field is applied, the curves shift towards higher intensities. Both HSPS and TPS have the largest upshift in $E_{\mathrm{wf}}$ for $\lambda=1.1 \mu \mathrm{m}$.
The amplitude of $E_{\text {wf }}$ in terms of $n_{0}$ for different values of laser pulse durations 30,40 and $50 \mathrm{fs}$ is plotted in Figs. 3(a-f), respectively. It is evidently observed that as $\tau$ increases, the maximum of $E_{\mathrm{wf}}$ declines and occurs at lower $n_{0}$ values. This behaviour is in complete agreement with Fig. 3(a) in Askari and Shahidani [34]. The maximum values of $E_{\text {wf }}$ for different $\tau$ values are shown in Table 2 . Applying magnetic field in this case also causes $E_{\mathrm{wf}}$ to shift to higher intensities. The $E_{\mathrm{wf}}$ amplitude experiences the largest increase for $\tau=40$ fs.

\section{Effect of laser pulse duration}

Another important parameter governing wake field excitation is the laser pulse duration. So, the amplitude of $E_{\mathrm{wf}}$ in terms of $\tau$ is shown in Figs. $4(\mathrm{a}-\mathrm{f})$ for $n_{0}=1 \times 10^{24} \mathrm{~m}^{-3}, 5 \times 10^{24} \mathrm{~m}^{-3}$ and $10 \times 10^{24} \mathrm{~m}^{-3}$ corresponding to $\lambda_{p}=33,15$ and $10.5 \mu \mathrm{m}$, respectively. It is observed that HSPS and TPS have a little different behaviour. For HSPS, the amplitude of $E_{\text {wf }}$ grows when $c \tau<\lambda_{p}$, peaks at $c \tau \approx \lambda_{p}$ and decreases when $c \tau>\lambda_{p}$. For TPS, the amplitude of $E_{\text {wf }}$ rises when $c \tau<0.5 \lambda_{p}$, hits the peak at $c \tau \approx 0.5 \lambda_{p}$ and plummets when $c \tau>0.5 \lambda$. The amplitude of $E_{\text {wf }}$ drops to its minimum at $c \tau \approx \lambda_{p}$ and remains unchanged until at $c \tau \approx 1.5 \lambda$ the amplitude rises again. What is more, for both HSPS and TPS cases, by increasing $n_{0}$ value, $E_{\text {wf }}$ reaches its maximum at lower $\tau$ values. This behaviour is in complete agreement with Fig. 3(b) in Askari and Shahidani [34]. The maximum values of $E_{\text {wf }}$ for different $n_{0}$ values are shown in Table 3. In the presence of magnetic field, the curves shift up and the maximum of $E_{\mathrm{wf}}$ happens at longer pulse durations for both HSPS and TPS.

\section{Effect of plasma frequency}

In Figs. 5( $(\mathrm{a}-\mathrm{f})$, the amplitude of $E_{\mathrm{wf}}$ in terms of $\omega_{p} / \omega_{L}$ is plotted for three different values of $s_{0}, 15$, 20 and $25 \mu \mathrm{m}$, respectively. It is concluded that with increasing the $\omega_{p} / \omega_{L}$ value, the amplitude of $E_{\mathrm{wf}}$ increases until it peaks at about $\omega_{p} / \omega_{L} \approx 1.15$ (HSPS) and 0.89 (TPS). The change of $s_{0}$ in this case has almost no effect on the behaviour and the intensity of $E_{\mathrm{wf}}$ The maximum values of $E_{\mathrm{wf}}$ for different $s_{0}$ values are shown in Table 4. Applying magnetic field causes the rise of magnitude $E_{\text {wf }}$ for both pulses, but the rise of TPS magnitude is more distinct than the HSPS one. 

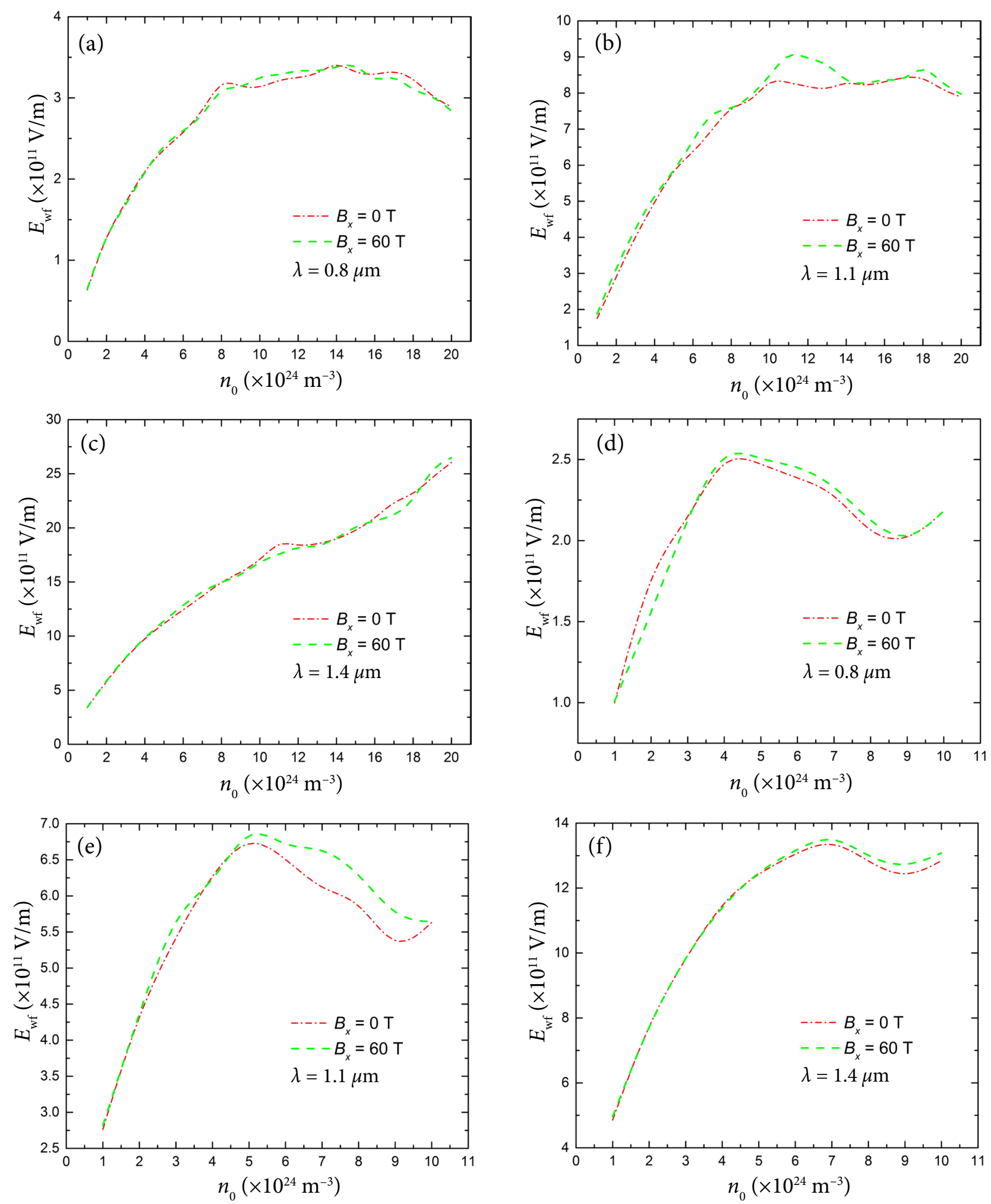

Fig. 2. Amplitude of wake field $E_{\mathrm{wf}}$ in terms of $n_{0}$ at (a) $\lambda=0.8 \mu \mathrm{m}$, (b) $\lambda=1.1 \mu \mathrm{m}$, (c) $\lambda=1.4 \mu \mathrm{m}$ for HSPS and at (d) $\lambda=0.8 \mu \mathrm{m}$, (e) $\lambda=1.1 \mu \mathrm{m}$ and (f) $\lambda=1.4 \mu \mathrm{m}$ for TPS.

\section{Effect of magnetic field}

Magnetic field has been an interesting issue in the study of laser plasma interaction. In order to have a better insight of the effect of magnetic field, the amplitude of $E_{\mathrm{wf}}$ in terms of $n_{0}$ is plotted for three values of longitudinal magnetic fields: $B_{x}=0$, 30 and $60 \mathrm{~T}$ in Figs. $6(\mathrm{a}, \mathrm{b})$ for HSPS and TPS, 

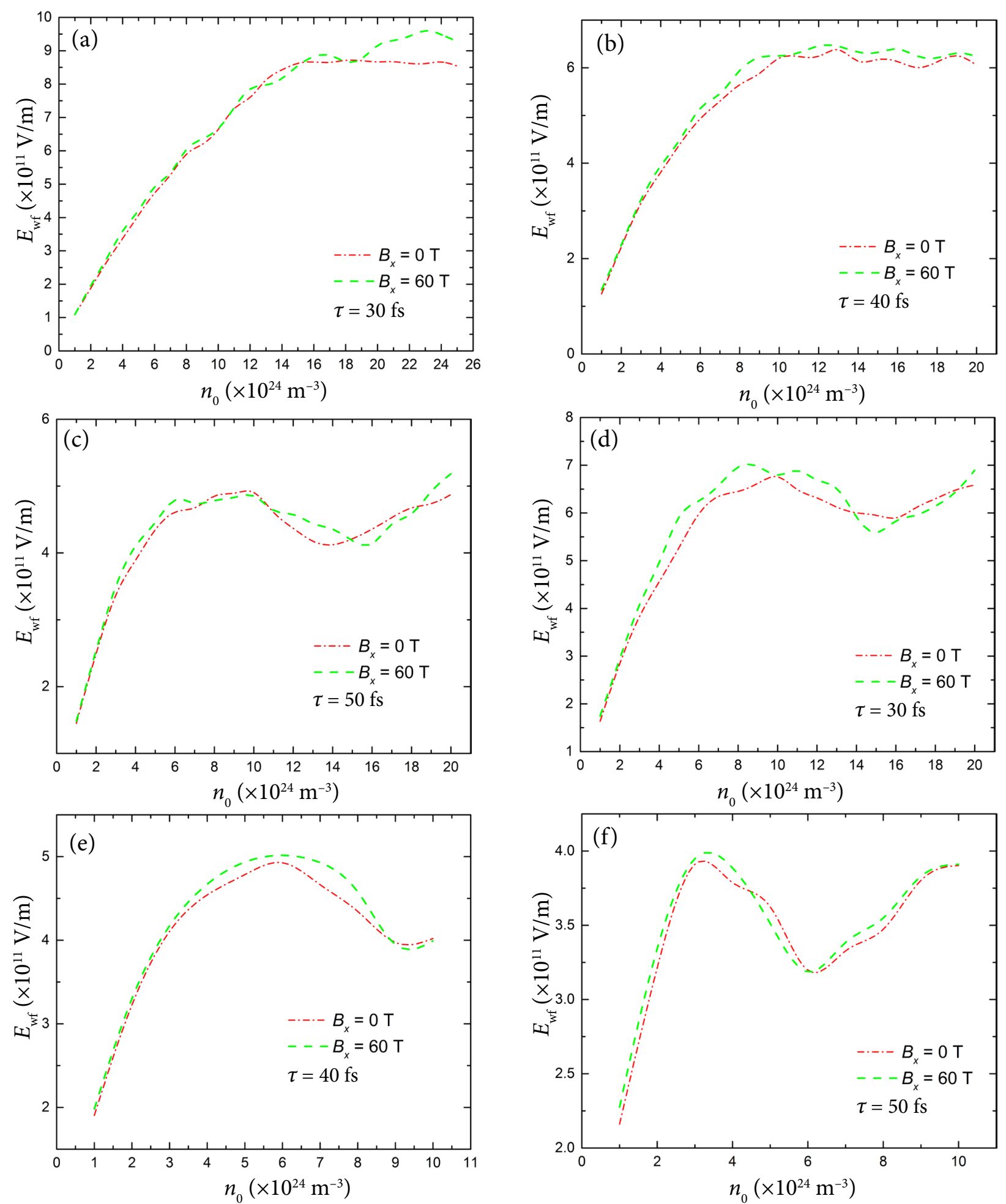

Fig. 3. Amplitude of wake field $E_{\mathrm{wf}}$ in terms of $n_{0}$ at (a) $\tau=30 \mathrm{fs}$, (b) $\tau=40 \mathrm{fs}$, (c) $\tau=50 \mathrm{fs}$ for HSPS and at (d) $\tau=30 \mathrm{fs}$, (e) $\tau=40 \mathrm{fs}$ and (f) $\tau=50$ fs for TPS.

Table 1. Maximum values of $E_{\mathrm{wf}}$ in terms of $n_{0}$ for three different $\lambda$ values.

\begin{tabular}{c|c|c|c|c}
\hline$\lambda, \mu \mathrm{m}$ & $\begin{array}{c}E_{\mathrm{wf}}^{\max }(\mathrm{HSPS}) \\
\left(\times 10^{11} \mathrm{~V} / \mathrm{m}\right)\end{array}$ & $n_{0}\left(\times 10^{24} \mathrm{~m}^{-3}\right)$ & $\begin{array}{c}E_{\mathrm{wf}}^{\max }(\mathrm{TPS}) \\
\left(\times 10^{11} \mathrm{~V} / \mathrm{m}\right)\end{array}$ & $n_{0}\left(\times 10^{24} \mathrm{~m}^{-3}\right)$ \\
\hline 0.8 & 3.454 & 14 & 2.557 & 4 \\
\hline 1.1 & 8.455 & 18 & 6.869 & 5 \\
\hline 1.4 & 26.044 & 20 & 13.545 & 7 \\
\hline
\end{tabular}


Table 2. Maximum values of $E_{\mathrm{wf}}$ in terms of $n_{0}$ for three different $\tau$ values.

\begin{tabular}{c|c|c|c|c}
\hline$\tau, \mathrm{fs}$ & $\begin{array}{c}E_{\mathrm{wf}}^{\max }(\mathrm{HSPS}) \\
\left(\times 10^{11} \mathrm{~V} / \mathrm{m}\right)\end{array}$ & $n_{0}\left(\times 10^{24} \mathrm{~m}^{-3}\right)$ & $\begin{array}{c}E_{\mathrm{wf}}^{\max }(\mathrm{TPS}) \\
\left(\times 10^{11} \mathrm{~V} / \mathrm{m}\right)\end{array}$ & $n_{0}\left(\times 10^{24} \mathrm{~m}^{-3}\right)$ \\
\hline 30 & 8.737 & 18 & 6.876 & 10 \\
\hline 40 & 6.514 & 13 & 5.037 & 6 \\
\hline 50 & 4.997 & 10 & 3.926 & 3 \\
\hline
\end{tabular}
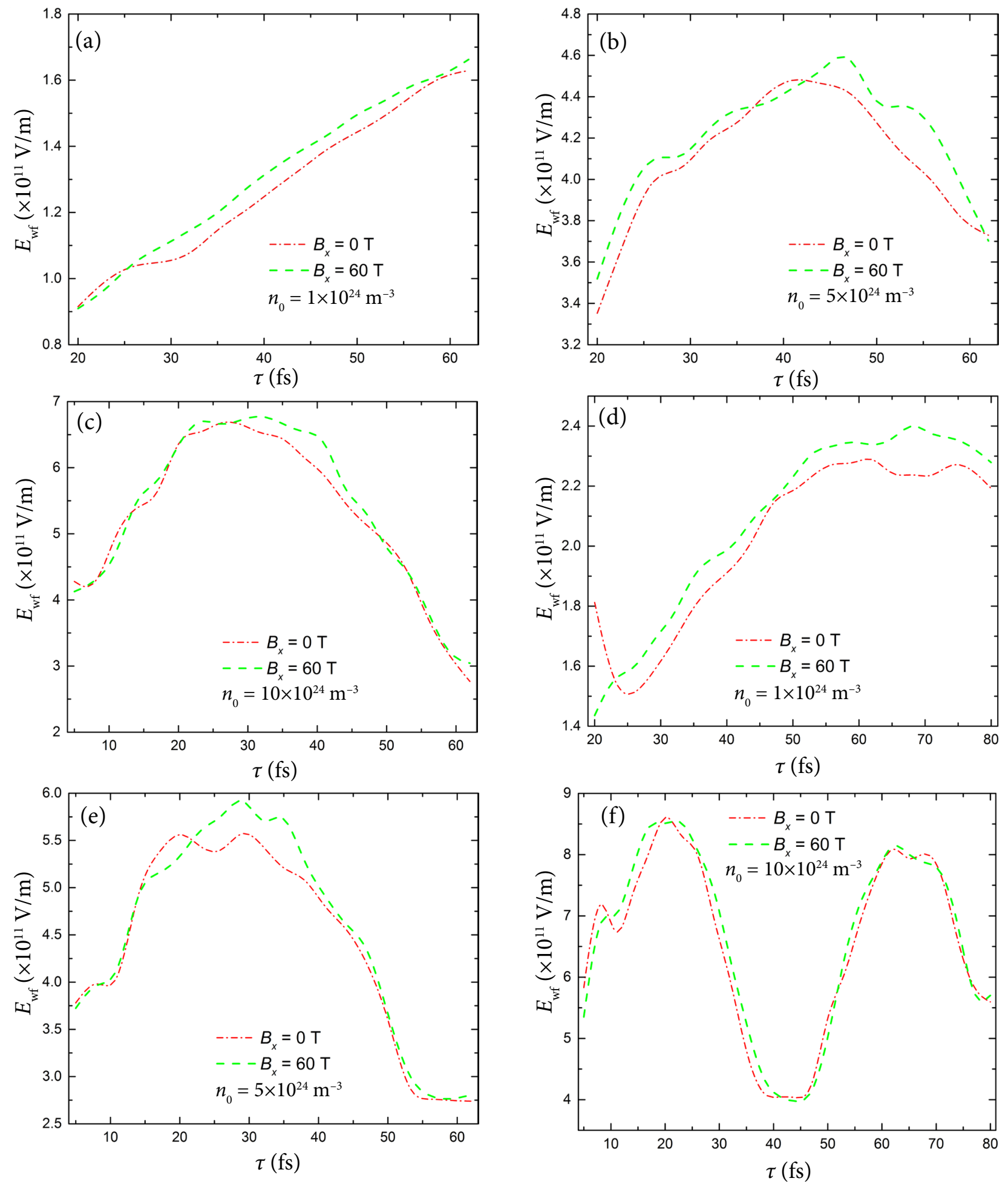

Fig. 4. Amplitude of wake field $E_{\mathrm{wf}}$ in terms of $\tau$ at (a) $n_{0}=1 \times 10^{24} \mathrm{~m}^{-3}$, (b) $n_{0}=5 \times 10^{24} \mathrm{~m}^{-3}$, (c) $n_{0}=10 \times 10^{24} \mathrm{~m}^{-3}$ for HSPS and at (d) $n_{0}=1 \times 10^{24} \mathrm{~m}^{-3}$, (e) $n_{0}=5 \times 10^{24} \mathrm{~m}^{-3}$ and (f) $n_{0}=10 \times 10^{24} \mathrm{~m}^{-3}$ for TPS. 
Table 3. Maximum values of $E_{\mathrm{wf}}$ in terms of $\tau$ for three different $n_{0}$ values.

\begin{tabular}{c|c|c|c|c}
\hline$n_{0}\left(\times 10^{24} \mathrm{~m}^{-3}\right)$ & $\begin{array}{c}E_{\mathrm{wf}}^{\max }(\mathrm{HSPS}) \\
\left(\times 10^{11} \mathrm{~V} / \mathrm{m}\right)\end{array}$ & $\tau, \mathrm{fs}$ & $\begin{array}{c}E_{\mathrm{wf}}^{\max }(\mathrm{TPS}) \\
\left(\times 10^{11} \mathrm{~V} / \mathrm{m}\right)\end{array}$ & $\tau, \mathrm{fs}$ \\
\hline 1 & 1.629 & 62 & 2.308 & 62 \\
\hline 5 & 4.500 & 41 & 5.659 & 29 \\
\hline 10 & 6.716 & 26 & 8.865 & 20 \\
\hline
\end{tabular}
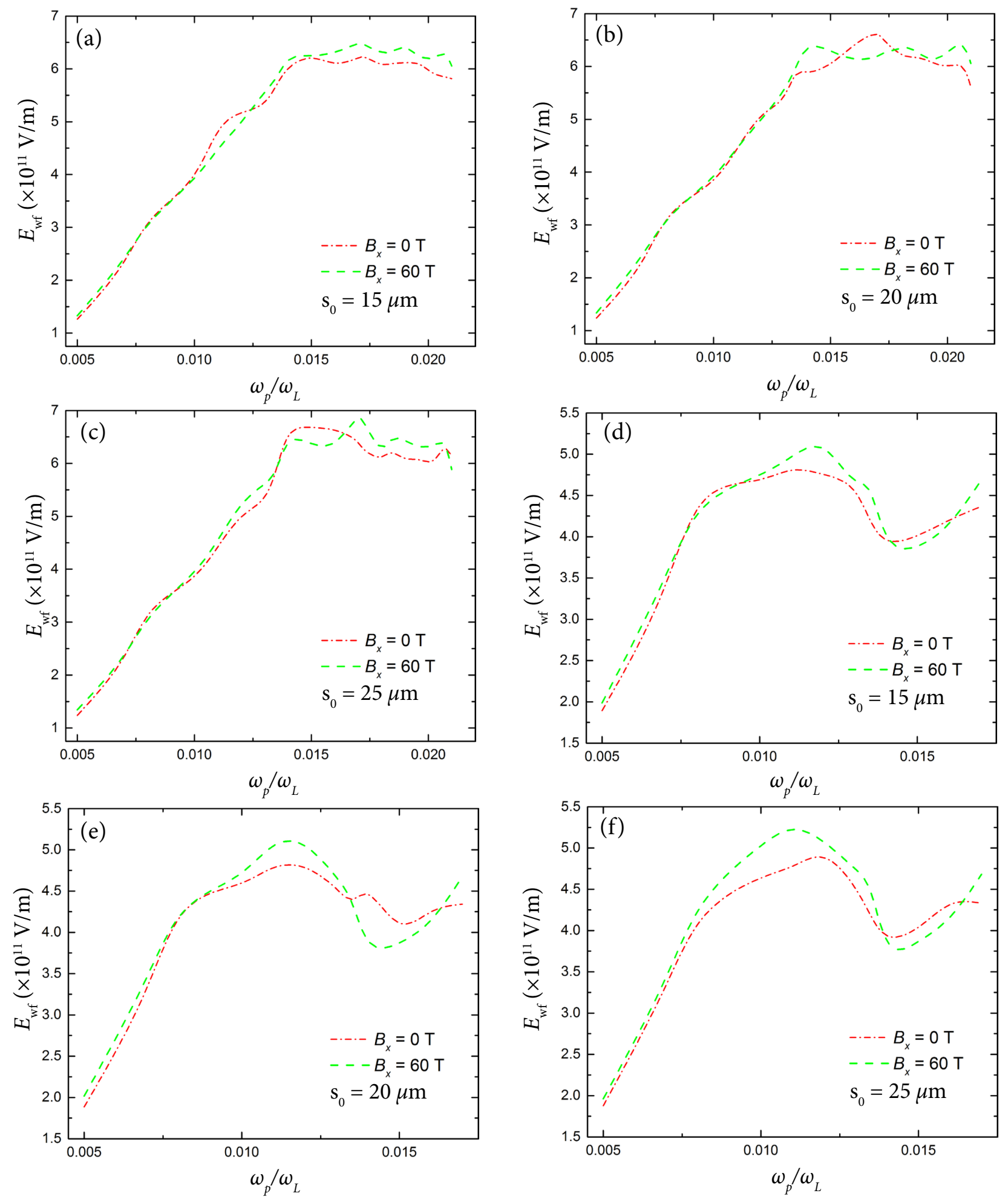

Fig. 5. Amplitude of wake field $E_{\text {wf }}$ in terms of $\omega_{p} / \omega_{L}$ at (a) $s_{0}=15 \mu \mathrm{m}$, (b) $\mathrm{s}_{0}=20 \mu \mathrm{m}$, (c) $\mathrm{s}_{0}=25 \mu \mathrm{m}$ for HSPS and at (d) $s_{0}=15 \mu \mathrm{m}$, (e) $s_{0}=20 \mu \mathrm{m}$ and (f) $s_{0}=25 \mu \mathrm{m}$ for TPS. 
Table 4. Maximum values of $E_{\mathrm{wf}}$ in terms of $\omega_{p} / \omega_{L}$ for three different $s_{0}$ values.

\begin{tabular}{c|c|c|c|c}
\hline$s_{0}, \mu \mathrm{m}$ & $\begin{array}{c}E_{\mathrm{wf}}^{\max }(\mathrm{HSPS}) \\
\left(\times 10^{11} \mathrm{~V} / \mathrm{m}\right)\end{array}$ & $\omega_{p} / \omega_{L}$ & $\begin{array}{c}E_{\mathrm{wf}}^{\max }(\mathrm{TPS}) \\
\left(\times 10^{11} \mathrm{~V} / \mathrm{m}\right)\end{array}$ & $\omega_{p} / \omega_{L}$ \\
\hline 15 & 6.278 & 1.15 & 4.857 & 0.81 \\
\hline 20 & 6.678 & 1.26 & 4.830 & 0.89 \\
\hline 25 & 6.705 & 1.09 & 4.997 & 0.89 \\
\hline
\end{tabular}
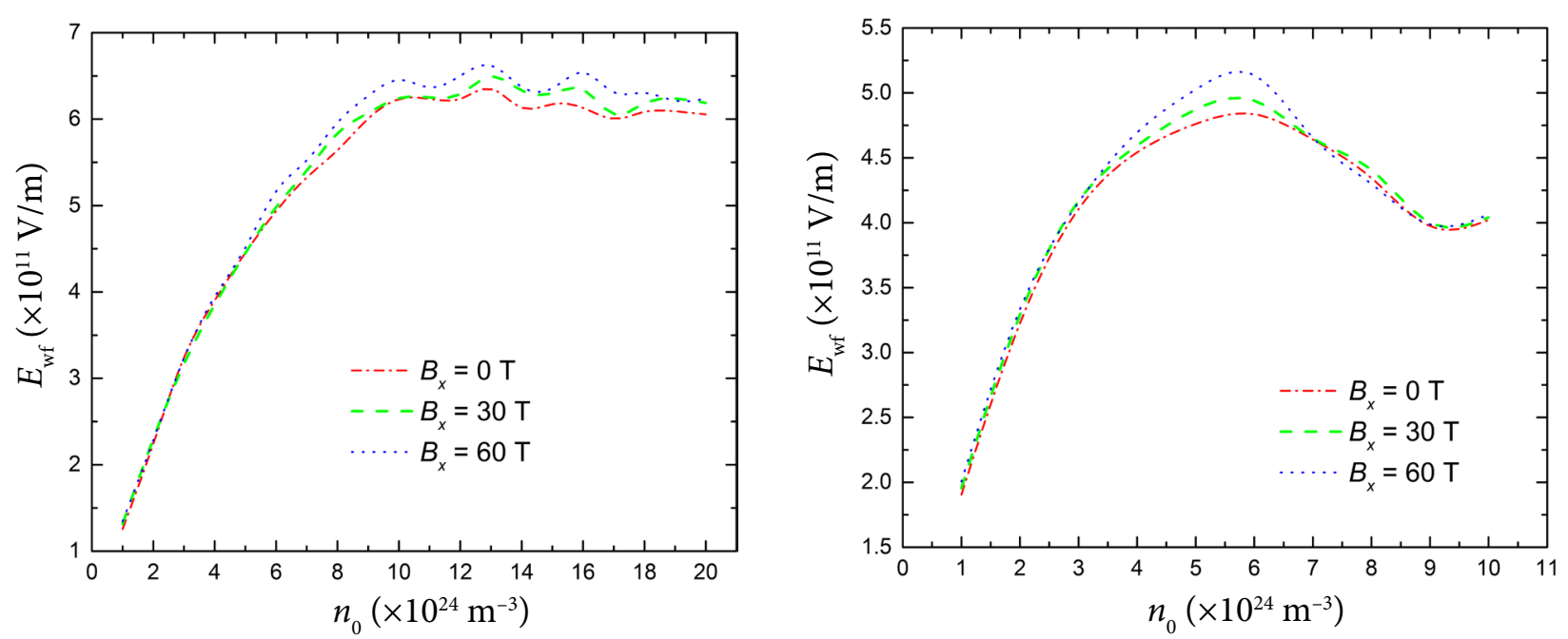

Fig. 6. Amplitude of wake field $E_{\mathrm{wf}}$ in terms of $n_{0}$ at $B_{x}=0,30$ and $60 \mathrm{~T}$ for (a) HSPS and (b) TPS.

Table 5. Maximum values of $E_{\mathrm{wf}}$ in terms of $n_{0}$ for three different $B_{x}$ values.

\begin{tabular}{c|c|c|c|c}
\hline$B_{x^{,}} \mathrm{T}$ & $\begin{array}{c}E_{\mathrm{wf}}^{\max }(\mathrm{HSPS}) \\
\left(\times 10^{11} \mathrm{~V} / \mathrm{m}\right)\end{array}$ & $n_{0}\left(\times 10^{24} \mathrm{~m}^{-3}\right)$ & $\begin{array}{c}E_{\mathrm{wf}}^{\max }(\mathrm{TPS}) \\
\left(\times 10^{11} \mathrm{~V} / \mathrm{m}\right)\end{array}$ & $n_{0}\left(\times 10^{24} \mathrm{~m}^{-3}\right)$ \\
\hline 0 & 6.473 & 13 & 4.900 & 6 \\
\hline 30 & 6.614 & 13 & 5.037 & 6 \\
\hline 60 & 6.727 & 13 & 5.300 & 6 \\
\hline
\end{tabular}

respectively. It is observed that for both pulses, when $B_{x}$ increases, the magnitude of $E_{\text {wf }}$ increases as well. This behaviour is not seen in Fig. 5(b) in Askari and Shahidani [34]. However, the trend of Figs. $6(a, b)$ is the same as that of Fig. $5(b)$ in Askari and Shahidani [34]. The maximum values of $E_{\text {wf }}$ for different $B_{x}$ values are shown in Table 5 .

\section{Conclusions}

In this paper, we have studied the effects of laser and plasma parameters on the wake field excitation with two different temporal pulse envelopes. For both shapes, the amplitude of $E_{\mathrm{wf}}$ increases when $\lambda$ rises at a definite $n_{0}$ value. Reversely, the $E_{\text {wf }}$ intensity declines when $\tau$ rises. We have also concluded that the behaviour of wake field differs in relation to the $n_{0}$ value for a separate $\tau$. For HSPS, the wake field intensity peaks at $c \tau \approx \lambda$, whereas the same occurs for TPS at $c \tau \approx 0.5 \lambda_{p}^{p^{2}}$. Moreover, the maximum of $E_{\mathrm{wf}}$ intensity has bigger magnitudes for higher $n_{0}$ in the studied range of $n_{0}$ values. Our study has shown that when $\omega_{p} / \omega_{L}$ value increases, the amplitude of $E_{\text {wf }}$ increases for HSPS and TPS until it reaches its climax for a distinct $\omega_{p} / \omega_{L}$ value. It has been clearly observed that applying a longitudinal magnetic field enhances wake field excitation. It is shown in Figs. 2, 3, 5 and 6 that the maximum value of $E_{\mathrm{wf}}$ for the same initial laser and plasma parameters is larger for the HSPS case compared to the TPS case whereas in Fig. 4 the highest value of $E_{\mathrm{wf}}$ for the TPS case is larger than for the HSPS case. Our results are compatible with the results presented by Askari and Shahidani [34]. 


\section{References}

[1] T.Y. Chien, C.L. Chang, C.H. Lee, J.Y. Lin, J. Wang, and S.Y. Chen, Spatially localized selfinjection of electrons in a self-modulated laserwakefield accelerator by using a laser-induced transient density ramp, Phys. Rev. Lett. 94(11), 115003 (2005), http://dx.doi.org/10.1103 PhysRevLett.94.115003

[2] D. Close, C. Giuliano, R. Hellwarth, L. Hess, F. McClung, and W. Wagner, 8A2 - The self-focusing of light of different polarizations, IEEE J. Quantum Electron. 2(9), 553-557 (1966), http:// dx.doi.org/10.1109/JQE.1966.1074077

[3] E. Esarey, P. Sprangle, J. Krall, and A. Ting, Overview of plasma-based accelerator concepts, IEEE Trans. Plasma Sci. 24(2), 252-288 (1996), http://dx.doi.org/10.1109/27.509991

[4] H. Lin, Z. Xu, L.-M. Chen, and J.C. Kieffer, Laser wakefield and self-modulation of driving pulse, Phys. Plasmas 10(8), 3371-3376 (2003), http:// dx.doi.org/10.1063/1.1588639

[5] J. Faure, Y. Glinec, A. Pukhov, S. Kiselev, S. Gordienko, E. Lefebvre, J.P. Rousseau, F. Burgy, and V. Malka, A laser-plasma accelerator producing monoenergetic electron beams, Nature 431 (7008), 541-544 (2004), http:/ dx.doi.org/10.1038/nature02963

[6] C.G.R. Geddes, Cs. Toth, J.van Tilborg, E. Esarey, C.B. Schroeder, D. Bruhwiler, C. Nieter, J. Cary, and W.P. Leemans, High-quality electron beams from a laser wakefield accelerator using plasmachannel guiding, Nature 431 (7008), 538-541 (2004), http://dx.doi.org/10.1038/nature02900

[7] S.P.D. Mangles, C.D. Murphy, Z. Najmudin, A.G.R. Thomas, J.L. Collier, A.E. Dangor, E.J. Divall, P.S. Foster, J.G. Gallacher, C.J. Hooker, et al., Monoenergetic beams of relativistic electrons from intense laser-plasma interactions, Nature 431 (7008), 535-538 (2004), http://dx.doi. org/10.1038/nature02939

[8] T. Tajima and J.M. Dawson, Laser electron accelerator, Phys. Rev. Lett. 43(4), 267-270 (1979), http://dx.doi.org/10.1103/PhysRevLett.43.267

[9] J.B. Rosenzweig, D.B. Cline, B. Cole, H. Figueroa, W. Gai, R. Konecny, J. Norem, P. Schoessow, and J. Simpson, Experimental observation of plasma wake-field acceleration, Phys. Rev. Lett. 61(1), 98-101 (1988), http://dx.doi.org/10.1103/ PhysRevLett.61.98

[10]C.E. Clayton, M.J. Everett, A. Lal, D. Gordon, K.A. Marsh, and C. Joshi, Acceleration and scattering of injected electrons in plasma beat wave accelerator experiments, Phys. Plasmas 1(5), 17531760 (1994), http://dx.doi.org/10.1063/1.870679

[11]M. Everett, A. Lal, D. Gordon, C.E. Clayton, K.A. Marsh, and C. Joshi, Trapped electron acceleration by a laser-driven relativistic plasma wave, Nature 368 (6471), 527-529 (1994), http://dx.doi. org/10.1038/368527a0

[12]Y. Kitagawa, T. Matsumoto, T. Minamihata, K. Sawai, K. Matsuo, K. Mima, K. Nishihara, H. Azechi, K.A. Tanaka, H. Takabe, and S. Nakai, Beat-wave excitation of plasma wave and observation of accelerated electrons, Phys. Rev. Lett. 68(1), 48-51 (1992), http://dx.doi.org/10.1103 PhysRevLett.68.48

[13]L.M. Gorbunov, N.E. Andreev, V.I. Kirsanov, A.A. Pogosova, and R.R. Ramazashvili, Resonant excitation of wakefields by a laser pulse in a plasma, JETP Lett. 55(10), 571-576 (1992), https:// www.researchgate.net/profile/NE_Andreev/ publication/253345212_Resonant_excitation_ of_wakefields_by_a_laser_pulse_in_a_plasma/ links/5501b8750cf231de076b21aa.pdf

[14]T.M. Antonsen and P. Mora, Self-focusing and Raman scattering of laser pulses in tenuous plasmas, Phys. Rev. Lett. 69(15), 2204-2207 (1992), http://dx.doi.org/10.1103/PhysRevLett.69.2204

[15]E. Esarey, J. Krall, and P. Sprangle, Envelope analysis of intense laser pulse self-modulation in plasmas, Phys. Rev. Lett. 72(18), 2887-2890 (1994), http://dx.doi.org/10.1103/PhysRevLett.72.2887

[16]K. Nakajima, Plasma-wave resonator for particle-beam acceleration, Phys. Rev. A 45(2), 1149-1156 (1992), http://dx.doi.org/10.1103 PhysRevA.45.1149

[17]J. Faure, C. Rechatin, A. Norlin, A. Lifschitz, Y. Glinec, and V. Malka, Controlled injection and acceleration of electrons in plasma wakefields by colliding laser pulses, Nature 444 (7120), 737-739 (2006), http://dx.doi.org/10.1038/nature05393

[18]W.P. Leemans, B. Nagler, A.J. Gonsalves, Cs. Toth, K. Nakamura, C.G.R. Geddes, E. Esarey, 
C.B. Schroeder, and S.M. Hooker, GeV electron beams from a centimetre-scale accelerator, Nat. Phys. 2(10), 696-699 (2006), http://dx.doi. org/10.1038/nphys418

[19]R. Wagner, S.Y. Chen, A. Maksimchuk, and D. Umstadter, Electron acceleration by a laser wakefield in a relativistically self-guided channel, Phys. Rev. Lett. 78(16), 3125-3128 (1997), http:// dx.doi.org/10.1103/PhysRevLett.78.3125

[20] B. Yan, N.A. Schultz, A.L. Efros, and P.C. Taylor, Universal distribution of residual carriers in tetrahedrally coordinated amorphous semiconductors, Phys. Rev. Lett. 84(18), 4180-4183 (2000), http://dx.doi.org/10.1103/PhysRevLett.84.4180

[21] M. Borghesi, A.J. MacKinnon, A.R. Bell, R. Gaillard, and O. Willi, Megagauss magnetic field generation and plasma jet formation on solid targets irradiated by an ultraintense picosecond laser pulse, Phys. Rev. Lett. 81(1), 112-115 (1998), http://dx.doi.org/10.1103 PhysRevLett.81.112

[22]R.N. Sudan, Mechanism for the generation of $10^{9} \mathrm{G}$ magnetic fields in the interaction of ultraintense short laser pulse with an overdense plasma target, Phys. Rev. Lett. 70(20), 3075-3078 (1993), http://dx.doi.org/10.1103/PhysRevLett.70.3075

[23]A. Lagutin, K. Rosseel, F. Herlach, J. Vanacken, and Y. Bruynseraede, Development of reliable 70 T pulsed magnets, Meas. Sci. Technol. 14(12), 2144 (2003), http://dx.doi.org/10.1088/0957. $0233 / 14 / 12 / 015$

[24]P.K. Shukla, G. Brodin, M. Marklund, and L. Stenflo, Wake field generation and nonlinear evolution in a magnetized electron-positron-ion plasma, Phys. Plasmas 15(8), 082305 (2008), http://dx.doi.org/10.1063/1.2970098

[25] H. Xiong, S. Liu, J. Liao, and X. Liu, Self-focusing of laser pulse propagating in magnetized plasma, Optik 121(18), 1680-1683 (2010), http://dx.doi. org/10.1016/j.ijleo.2009.03.019
[26] V.B. Krasovitskii, V.G. Dorofeenko, V.I. Sotnikov, and B.S. Bauer, Interaction of powerful laser pulse with magnetized plasma, Phys. Plasmas 11(2), 724-742 (2004), http://dx.doi. org/10.1063/1.1633556

[27]P. Jha, R.K. Mishra, A.K. Upadhyay, and G. Raj, Spot-size evolution of laser beam propagating in plasma embedded in axial magnetic field, Phys. Plasmas 14(11), 114504 (2007), http://dx.doi. org/10.1063/1.2815789

[28] C.K. Birdsall and A.B. Langdon, Plasma Physics via Computer Simulation (McGraw-Hill, 1985), http:// books.google.com/books?id=7TMbAQAAIAA)

[29] H.C. Wu, JPIC \& How to Make a PIC Code (Cornell University Library, 2011), http://arxiv. org/abs/1104.3163

[30]E. Esarey and C.B. Schroeder (2003), http://oskicat.berkeley.edu/record=b20937049 S1

[31]C. Huang, V.K. Decyk, C. Ren, M. Zhou, W. Lu, W.B. Mori, J.H. Cooley, T.M. Antonsen Jr., and T. Katsouleas, QUICKPIC: A highly efficient particle-in-cell code for modeling wakefield acceleration in plasmas, J. Comput. Phys. 217(2), 658-679 (2006), http://dx.doi.org/10.1016/j.jcp.2006.01.039

[32]K. Yee, Numerical solution of initial boundary value problems involving Maxwell's equations in isotropic media, IEEE Trans. Antennas Propag. 14, 302-307 (1966), http://dx.doi.org/10.1109 TAP.1966.1138693

[33]J.P. Boris, Relativistic plasma simulation-optimization of a hybrid code, in: Proceedings of Fourth Conference on Numerical Simulations of Plasmas (Naval Research Laboratory, Washington, D. C., 1970) pp. 3-67, http://www.dtic.mil/dtic/tr/fulltext/u2/a023511.pdf

[34] H.R. Askari and A. Shahidani, Influence of properties of the Gaussian laser pulse and magnetic field on the electron acceleration in laser-plasma interactions, Opt. Laser Technol. 45, 613-619 (2013), http://dx.doi.org/10.1016/j.optlastec.2012.05.023

\title{
PLIŪPSNIO LAUKO ŽADINIMO, INTENSYVIU LAZERIU ŠVITINANT MAGNETIZUOTĄ PLAZMĄ, MODELIS: SINUSO PUSPERIODŽIO IR TRAPECINĖ IMPULSO FORMOS
}

\author{
A. Rahimian, H. Zahed \\ Sahand technologijos universitetas, Tabrizas, Iranas
}

